\title{
Efficacy of Neuroprotective Drugs in Acute Ischemic Stroke: Is It Helpful?
}

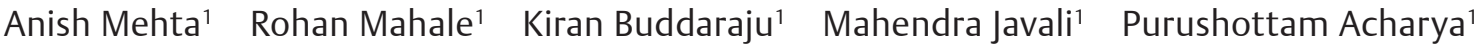 \\ Rangasetty Srinivasa ${ }^{1}$ \\ ${ }^{1}$ Department of Neurology, Ramaiah Medical College and Hospital, \\ Address for correspondence Anish Mehta, DM, Department \\ of Neurology, Ramaiah Medical College and Hospital, Bengaluru \\ 560 054, Karnataka, India (e-mail: anishmehta1302@gmail.com).
}

J Neurosci Rural Pract 2019;10:576-581

\author{
Abstract \\ Keywords \\ - acute ischemic stroke \\ - cerebrolysin \\ - citicoline \\ - edaravone \\ - middle cerebral artery \\ - minocycline \\ - neuroprotective \\ agents
}

Background Out of several neuroprotective drugs (NPDs) studied in animals and humans, four NPDs (citicoline, edaravone, cerebrolysin, and minocycline) have been found to have beneficial effects in acute ischemic stroke (AIS).

Objective The purpose is to evaluate the efficacy of citicoline, edaravone, minocycline, and cerebrolysin compared with placebo in patients with middle cerebral artery (MCA) territory AIS.

Materials and Methods This was a prospective, single center, single-blinded, and hospital-based study. One hundred patients with MCA territory AIS with 20 patients in each group including control group were included. Barthel index (BI), National Institute of Health Stroke Scale (NIHSS) score, and modified Rankin Scale score were recorded at admission, at day 11 and after 90 days.

Results The mean NIHSS score was significantly lesser at day 11 and after 90 days in citicoline, edaravone, and cerebrolysin group in comparison with placebo. Similarly, the mean BI score was significantly higher at day 11 and after 90 days in citicoline, edaravone, and cerebrolysin group in comparison with placebo. In minocycline group, there was no significant change in the NIHSS score and BI score at day 11 and after 90 days.

Conclusion There was significant improvement in the functional outcome of patients with AIS involving MCA territory at 90 days receiving citicoline, edaravone, and cerebrolysin. However, minocycline did not offer the same efficacy as compared with other neuroprotective agents.

\section{Introduction}

An acute global or focal neurological deficit lasting longer than 24 hours or leading to death and which is of no etiology other than vascular is termed as stroke. ${ }^{1}$ It is one of the major causes of death and it causes significant morbidity including physical dependence, cognitive decline, depression, and seizures. Intravenous recombinant tissue plasminogen activator (rt-PA; alteplase) is the only approved medical treatment for acute ischemic stroke (AIS) that helps in the recanalization of the occluded arteries and improves functional outcome. ${ }^{2}$ The treatment of AIS includes intravenous thrombolytics, blood sugar optimization, temperature control, blood pressure control, reduction in raised intracranial pressure, and neuroprotective drugs (NPDs). ${ }^{3}$ About 2 to $5 \%$ of cases are eligible to receive rt-PA treatment. ${ }^{4}$ This has fueled the interest in the development of neuroprotective therapies. The present study was conducted to determine the efficacy of four NPD: citicoline, edaravone, cerebrolysin, and minocycline, with placebo comparison in patients with middle cerebral artery (MCA) territory AIS.

\section{Materials and Methods}

This was a prospective, single-blinded, hospital-based study. The study period was from December 2014 to December 2017 with approval by the institutional ethics committee. Consecutive patients with age $>18$ years having focal 
neurological deficits presenting with magnetic resonance imaging (MRI) brain or computed tomography (CT) head suggestive of MCA territory AIS presenting within 24 hour of symptom onset, National Institute of Health Stroke Scale (NIHSS) score of $>4$ and with a modified Rankin Scale (mRS) score of 0 or 1 before the stroke were included in this study. Patients with hemorrhagic stroke, brainstem and cerebellar strokes, transient ischemic attacks, brain tumor, demyelinating diseases, inflammatory diseases, craniotomies, traumatic brain injuries, hepatic failure, congestive heart failure, acute myocardial infarction, pregnancy and lactation, systemic malignancy, acute or chronic renal failure, and known allergy to above group of drugs/tetracycline group of drugs were excluded from the study.

\section{Intervention}

Hundred patients were recruited for the study. Five groups of patients were compared. Informed written consent was taken. Patients in each group received $150 \mathrm{mg}$ aspirin. The control group $(\mathrm{N})$ received supportive care. Each group had 20 patients of either gender. The drug citicoline is an exogenous form of cytidine-5'-diphosphocholine needed for membrane biosynthesis. It acts as a membrane stabilizer and reduces free radical formation thus reducing ischemic injury. ${ }^{5}$ The citicoline dose used was $500 \mathrm{mg}$ twice daily for 6 weeks either as parenteral or oral formulation. Edaravone is also free radical scavenger that delays neuronal death, prevents vascular endothelial injury, inhibits in vitro lipoxygenase pathway activation in the arachidonic acid cascade and phosphatidylcholine liposomal membrane peroxidation, thereby reducing ischemic brain edema. ${ }^{6}$ It was administered as intravenous infusion at a dosage of $30 \mathrm{mg}$ twice daily over 60 minutes for 14 days. Minocycline is lipophilic drug that crosses the blood-brain barrier (BBB), inhibits microglial activation, reduce $\mathrm{T}$ cell migration and reduces neuronal apoptosis and generation of free radicals and chemokines and their receptors expression in the central nervous system. ${ }^{7}$ Minocycline was administered as oral formulation in a dose of $200 \mathrm{mg} /$ day for 10 days. The drug cerebrolysin is a porcine brain-derived free amino acids and low-molecular-weight neuropeptides $(10 \mathrm{kDa}){ }^{8}$ Cerebrolysin was administered as intravenous infusion in a dose of $30 \mathrm{~mL}$ diluted in $100 \mathrm{~mL}$ saline over 60 minutes for 10 days.

\section{Clinical Characteristics}

The demographic details, clinical symptomatology, presence of diabetes mellitus, hypertension, ischemic heart disease, dyslipidemia, atrial fibrillation, rheumatic heart disease, alcohol, and nicotine abuse, time of onset of symptoms to NPA administration, if thrombolytics was used and NIHSS at admission, at day 11 and after 90 days, were recorded. The location of infarct (right or left) and Alberta Stroke Program Early CT score (ASPECTS) score was calculated in each patient.

\section{Investigations}

The following blood investigations were performed in all patients that include complete blood counts, prothrombin time and international normalized ratio, activated plasma thromboplastin time, renal function test, serum electrolytes, random blood sugar, glycated hemoglobin, fasting lipid profile, serum homocysteine, human immunodeficiency virus, hepatitis B virus, and syphilis serology. MRI brain including diffusion-weighted imaging in 1.5-Tesla and CT brain were done in every patient.

\section{Outcome}

The clinical assessment to record the functional outcome was done at discharge and 90 days. Barthel index (BI) and mRS were employed to assess the functional outcome. The $\mathrm{mRS}$ score and BI were recorded at admission, day 11 and after 90 days. The hospital mortality and its causes were also included.

\section{Statistical Analysis}

Student's unpaired $t$-test was used to compare continuous variables expressed as mean \pm standard deviation. Chisquared test was used to compare categorical variables expressed as frequency with percentage. Analysis of variance tests and multiple comparison tests were used for comparison of NIHSS, mRS, and BI scores among individual NPA and placebo group. Mann-Whitney U tests were used to determine a significant change in mRS score at day 11 and after 90 days. Kruskal-Wallis tests were used to determine the existence of statistically significant differences among different groups with change in mRS score at day 11 and after 90 days. $p$-Value $\leq 0.05$ was considered as statistically significant. The statistical analyses were conducted using SPSS 17 software (IBM. Inc., Texas, United States).

\section{Results}

One hundred patients were included in the study with 59 males and 41 females. The mean age of the patients was $58.4 \pm 7.2$ (range: 50-67) years. They were 20 patients each in citicoline $(\mathrm{C})$, edaravone $(\mathrm{E})$, minocycline $(\mathrm{M})$, cerebrolysin $(\mathrm{Cs})$, and fifth control group $(\mathrm{N})$. There were 12 males and 8 females in citicoline group; 11 males and 9 females in edaravone group, 13 males and 7 females in minocycline group, 12 males and 8 females in cerebrolysin group, and 11 males and 9 females in control group. Each group was matched for age, gender, time of initiation of treatment, preexisting medical conditions, mRS/NIHSS, and ASPECTS score at the time of admission. The mean time until admission after onset of stroke symptoms and meantime until treatment with NPA administration was similar among all the groups with no statistical significance. Nineteen patients received thrombolysis treatment with no statistically significant difference among the groups. The demographic details and clinical profile of the three groups are shown in - Table 1.

There was reduction in mean NIHSS score at day 11 and after 90 days in citicoline, edaravone, and cerebrolysin group in comparison with placebo assuming statistical significance (-Table 2). However, in minocycline group, the NIHSS score did not reduce at day 11 and after 90 days. Similarly, the mean BI score was significantly higher at day 11 and after 90 days in citicoline, edaravone, and cerebrolysin group in comparison with placebo (-Table 3 ). 
Table 1 Baseline characteristics of the five treatment groups in the trial $(\boldsymbol{n}=100)$

\begin{tabular}{|c|c|c|c|c|c|c|}
\hline Characteristics & $\begin{array}{l}\text { Citicoline }(n=20) \text {, } \\
n(\%)\end{array}$ & $\begin{array}{l}\text { Edaravone } \\
(n=20), n(\%)\end{array}$ & $\begin{array}{l}\text { Minocycline, } \\
(n=20), n \\
(\%)\end{array}$ & $\begin{array}{l}\text { Cerebrolysin } \\
(n=20), n(\%)\end{array}$ & $\begin{array}{l}\text { Placebo } \\
(n=20), n(\%)\end{array}$ & $p$-Value \\
\hline Mean age (SD) & 59.5 & 57.3 & 58.8 & 61.9 & 64.9 & 0.34 \\
\hline Gender (male) & $12(60)$ & $11(55)$ & $13(65)$ & $12(60)$ & $11(55)$ & 0.57 \\
\hline Time until admission (h) & $11.59 \pm 3.25$ & $11.10 \pm 3.60$ & $10.60 \pm 3.84$ & $11.50 \pm 3.75$ & $11.81 \pm 3.30$ & 0.76 \\
\hline Time until treatment (h) & $13.47 \pm 4.34$ & $13.1 \pm 4.10$ & $12.40 \pm 3.87$ & $12.94 \pm 4.45$ & $13.30 \pm 4.95$ & 0.64 \\
\hline $\begin{array}{l}\text { Dominant lobe MCA } \\
\text { infarction }\end{array}$ & $11(55)$ & $12(60)$ & $12(60)$ & $12(60)$ & $11(55)$ & 0.24 \\
\hline HTN & $11(55)$ & $13(65)$ & $13(65)$ & $12(60)$ & $11(55)$ & 0.83 \\
\hline DM & $7(35)$ & $7(35)$ & $7(35)$ & $8(40)$ & $8(40)$ & 0.25 \\
\hline IHD & $5(25)$ & $6(30)$ & $5(25)$ & $6(30)$ & $5(25)$ & 0.30 \\
\hline Smoking & $6(30)$ & $5(25)$ & $3(15)$ & $4(20)$ & $6(30)$ & 1.86 \\
\hline Alcohol & $4(20)$ & $3(15)$ & $4(20)$ & $2(10)$ & $4(20)$ & 2.53 \\
\hline Thrombolysis treatment & $4(20)$ & $4(20)$ & $3(15)$ & $4(20)$ & $4(20)$ & 0.26 \\
\hline ASPECTS score (median) & 8 & 7 & 7 & 8 & 7 & 0.42 \\
\hline NIHSS score & $14 \pm 4.34$ & $13.25 \pm 5.3$ & $13.1 \pm 4.59$ & $14.15 \pm 5.30$ & $13.35 \pm 4.53$ & 0.98 \\
\hline Bl score & $36 \pm 3.83$ & $37.5 \pm 4.25$ & $31 \pm 3.93$ & $35 \pm 4.51$ & $38 \pm 4.03$ & 0.76 \\
\hline mRS score & $4.5 \pm 0.17$ & $3.75 \pm 0.19$ & $4.2 \pm 0.21$ & $4.3 \pm 0.20$ & $4.45 \pm 0.15$ & 0.06 \\
\hline
\end{tabular}

Abbreviations: ASPECTS, Alberta Stroke Program Early CT score; BI, Barthel Index; DM, diabetes mellitus; HTN, hypertension; IHD, ischemic heart disease; MCA, middle cerebral artery; mRS, modified Rankin Scale; NIHSS, National Institute of Health Stroke Scale; SD, standard deviation.

Table 2 Comparison of National Institute of Health Stroke Scale scores among the five groups pre- and post-therapy

\begin{tabular}{|c|c|c|c|c|}
\hline \multirow[t]{2}{*}{ Groups } & \multirow[t]{2}{*}{$n$} & \multirow[t]{2}{*}{ Pretherapy } & \multicolumn{2}{|c|}{ Post-therapy } \\
\hline & & & Day 11 & 90 days \\
\hline Citicoline & 20 & $14.00 \pm 4.34$ & $8.90 \pm 4.21^{1, *}$ & $3.53 \pm 2.22^{\mathrm{a}, *}$ \\
\hline Edaravone & 20 & $13.25 \pm 5.33$ & $6.84 \pm 3.60^{2, *}$ & $2.94 \pm 1.95^{\mathrm{b}, *}$ \\
\hline Minocycline & 20 & $13.10 \pm 4.59$ & $9.90 \pm 4.90^{3}$ & $4.82 \pm 2.41^{c}$ \\
\hline Cerebrolysin & 20 & $14.15 \pm 5.30$ & $9.05 \pm 4.90^{4, *}$ & $4.50 \pm 3.23^{d, *}$ \\
\hline Placebo & 20 & $13.35 \pm 4.53$ & $10.20 \pm 4.70$ & $5.82 \pm 2.90$ \\
\hline
\end{tabular}

Abbreviations: ANOVA, analysis of variance; SD, standard deviation.

Note: Values are expressed as mean \pm SD.

${ }^{1} p<0.001$ versus the placebo group by ANOVA.

${ }^{2} p<0.001$ versus the placebo group by ANOVA.

${ }^{3} p=0.90$ versus the placebo group by ANOVA.

${ }^{4} p=0.01$ versus the placebo group by ANOVA.

${ }^{a} p<0.001$ versus the placebo group by ANOVA.

${ }^{\mathrm{b}} \mathrm{p}<0.001$ versus the placebo group by ANOVA.

${ }^{c} p=0.29$ versus the placebo group by ANOVA.

${ }^{d} p=0.05$ versus the placebo group by ANOVA.

${ }^{*} p$-value significant.

\section{Safety Analysis}

The adverse effects of neuroprotective agents were recorded every day during the stay in hospital till day 11 . No adverse effects were noted in any of the group except two patients in cerebrolysin group who had a fever that resolved with antipyretics.

\section{Analyses of Mortality}

There were five deaths with one death in each group. The cause of death in edaravone and minocycline group was due to malignant MCA territory infarct with herniation (despite decompressive craniectomy). Other three deaths occurred following discharge within after 90 days (cause not known).

\section{Discussion}

Agents that are easy to administer, ability to reduce tissue damage during AIS, will help in improving functional outcomes and quality of life of patients. One of those agents is the neuroprotective agents. These agents protect the brain 
Table 3 Comparison of Barthel Index scores between the five groups and placebo pre- and post-therapy

\begin{tabular}{|l|l|l|l|l|}
\hline Groups & $n$ & Pretherapy & Post-therapy & 90 days \\
\cline { 3 - 5 } & & & Day 11 & $86.0 \pm 8.19^{\mathrm{a}, *}$ \\
\hline Citicoline & 20 & $36.0 \pm 17.13$ & $64.0 \pm 17.13^{1, *}$ & $88.88 \pm 6.18^{\mathrm{b}, *}$ \\
\hline Edaravone & 20 & $37.5 \pm 19.0$ & $68.68 \pm 12.75^{2, *}$ & $76.35 \pm 8.12^{\mathrm{c}}$ \\
\hline Minocycline & 20 & $31.0 \pm 17.59$ & $51.5 \pm 20.20^{3}$ & $78.33 \pm 12.83^{\mathrm{d}, *}$ \\
\hline Cerebrolysin & 20 & $35.0 \pm 20.19$ & $57.25 \pm 21.61^{4, *}$ & $74.82 \pm 10.83$ \\
\hline Placebo & 20 & $38.0 \pm 17.95$ & $54.25 \pm 21.10$ & \\
\hline
\end{tabular}

Abbreviations: ANOVA, analysis of variance; SD, standard deviation.

Note: Values are expressed as mean \pm SD.

${ }^{1} p<0.001$ versus the placebo group by ANOVA.

${ }^{2} p<0.001$ versus the placebo group by ANOVA.

${ }^{3} p=0.07$ versus the placebo group by ANOVA.

${ }^{4} p=0.03$ versus the placebo group by ANOVA.

${ }^{a} p<0.001$ versus the placebo group by ANOVA.

${ }^{\mathrm{b}} p<0.001$ versus the placebo group by ANOVA.

${ }^{c} p=0.06$ versus the placebo group by ANOVA.

${ }^{\mathrm{d}} p=0.02$ versus the placebo group by ANOVA.

${ }^{*} p$-value significant.

from ischemia in AIS. ${ }^{9}$ Antithrombotics, antiplatelets, and thrombolytics also produce neuroprotection by targeting the cerebral vessels and are considered as extrinsic or indirect neuroprotectants. Direct neuroprotectants act directly on the neuron. Brain ischemia causes disruption of the BBB and loss of microvascular integrity as it triggers extracellular and intracellular proteolytic cascades. ${ }^{10}$ During reperfusion, there is reactive oxygen species (ROS) production that causes ischemic cells to secrete inflammatory cytokines and chemokines that increase adhesion molecules and cause recruitment of peripheral leukocytes. The recruited inflammatory cells release more cytokines, matrix metalloproteinases (MMPs), nitric oxide, and more ROS. MMPs cause BBB disruption, activation of microglia, and recruitment of peripheral inflammatory cells. ${ }^{11}$ The pharmacological modulation of molecular targets in the ischemic cascade can produce neuroprotection. ${ }^{9}$ These targets include glutamate release and glutamate receptor activation, excitotoxicity, calcium influx into cells, mitochondrial dysfunction, and activation of intracellular enzymes, free radical production, nitric oxide production, apoptosis, and inflammation. We used four neuroprotective agents in our study. The objective of our study was to determine the effectiveness of four neuroprotective agents (citicoline, edaravone, minocycline, and cerebrolysin) in changing the functional outcome at 90 days of patients with AIS involving MCA territory. All the patients in our study had moderate-to-severe stroke with mean NIHSS score of 14 .

Minocycline is an antibiotic belonging to the tetracycline family and has anti-inflammatory and neuroprotective action. It is a ROS scavenger protecting brain tissue against oxidative stress, which inhibits microglial activation and caspase-1, caspase-3, cyclo-oxygenase-2, inducible nitric oxide synthase, p38 mitogen-activated protein kinase, and MMP-9. ${ }^{12}$ A study by Lampl et al, on minocycline usage in AIS, showed beneficial effect in minocycline-treated group with significant improvement in NIHSS score. ${ }^{13} \mathrm{~A}$ study by Fagan et al showed that with intravenous minocycline (no controls), $50 \%$ (60 patients) of patients had mRS of 0 or 1 at
90 days. ${ }^{14}$ A study by Padma Srivastava et al also showed significant improvement in NIHSS score, BI score, and mRS score with minocycline. ${ }^{15} \mathrm{~A}$ study by Amiri-Nikpour et al showed a similar decrease in NIHSS in patients receiving minocycline at 90 days. ${ }^{16}$ However, study by Kohler et al did not find any benefit with minocycline. ${ }^{17}$ The dose used was $100 \mathrm{mg} /$ day, half of what the other studies had used, and was continued only for 2.5 days. In our study, 20 patients received oral minocycline at a dosage of $200 \mathrm{mg} /$ day for 5 days similar to the above studies. The mean time between stroke onset and minocycline administration was 12.4 hours. In the other studies, it was from 5 to 24 hours. There was decrease in NIHSS score and improvement in BI score in minocycline-treated group and control group but was not statistically significant. Minocycline was not effective as compared with control in improving the functional outcome.

Cerebrolysin is a neuropeptide and has neuroprotective properties. It reduces excitotoxicity and inhibits free radical generation, microglial activation/neuroinflammation, and calpain activation/apoptosis. It has neurotrophic activity as it promotes neuronal sprouting, improves cellular survival, and stimulates neurogenesis. Cerebrolysin acute stroke treatment in Asia trial did not show a significant difference in the functional outcome between the cerebrolysin and placebo groups, but there was a beneficial trend in favor of cerebrolysin on post-hoc analysis. ${ }^{18}$ Cerebrolysin and recovery after stroke trial showed that patients treated with cerebrolysin had an improvement in the NIHSS on day 90 compared with placebo. ${ }^{19}$ Study by Xue et al on cerebrolysin in AIS showed significant improvement of 21-day NIHSS scores with cerebrolysin. ${ }^{20}$ In our study, 20 patients received intravenous cerebrolysin with mean baseline NIHSS score of $14.15 \pm 5.3$, and there was a significant improvement in the NIHSS and BI score at day 11 and after 90 days.

Edaravone is an antioxidant and is low molecular weight lipid-soluble free radical scavenger that readily crosses the BBB. Edaravone AIS Group (Otomo clinical trial report) showed that edaravone administration resulted 
in significant clinical improvement. ${ }^{21}$ A study by Sinha et al showed that patients in edaravone-treated group had significant reduction in NIHSS and improvement in BI at 90 days. ${ }^{22}$ Edaravone-Citicoline Comparative Study in AIS by Mitta et al-showed that edaravone usage resulted in a better outcome at 90 days in patients with AIS. ${ }^{23}$ In our study, 20 patients received intravenous edaravone with mean baseline NIHSS score of $13.2 \pm 5.3$ with significant improvement in the NIHSS and BI score at day 11 and after 90 days.

Citicoline helps in restoring the activity of mitochondrial ATPase and membrane $\mathrm{Na}^{+} / \mathrm{K}+$ ATPase, inhibits the activation of phospholipase $\mathrm{A} 2$, and accelerates the reabsorption of cerebral edema. ${ }^{24}$ The International Citicoline Trial on Acute Stroke study showed similar global recovery at 90 days in citicoline and placebo group. The study did not provide evidence that citicoline is efficacious in moderate-to-severe AIS. ${ }^{25}$ A meta-analysis by Saver ( 10 controlled clinical trials that used citicoline) showed that citicoline caused a significant decrease in the frequency of death or disability at follow-up. ${ }^{26}$ Study by Ghosh et al reported favorable outcome and an increased probability of complete recovery following citicoline treatment in all types of strokes. ${ }^{27}$ In our study, 20 patients received intravenous/oral citicoline with mean baseline NIHSS score of $14.0 \pm 4.3$ with significant improvement in the NIHSS and BI score at day 11 and after 90 days.

The small sample size in each group was the limitation of our study. It was a single-blinded study wherein the investigators were aware about the neuroprotective agents received by the patients and the outcome was assessed by the investigators themselves with a possibility of research bias. A randomized control trial with a bigger sample size is required to strengthen the results of this study.

\section{Conclusion}

Neuroprotective agents do help in patients with AIS, and we found that citicoline, edaravone, and cerebrolysin were effective in improving the functional outcome of patients with AIS involving MCA territory at 90 days. However, minocycline did not offer the same efficacy as compared with other neuroprotective agents.

\section{Funding}

None.

\section{Conflict of Interest}

None declared.

\section{Acknowledgments}

The authors would like to thank Dr. Shivaraj, Associate Professor, Department of Community Medicine, for his help in carrying out statistical analysis.

\section{References}

1 Odusote KO. Management of stroke. Niger Med Pract 1996;32:54-61
2 Adams HP Jr, del Zoppo G, Alberts MJ, et al; American Heart Association/American Stroke Association Stroke Council; American Heart Association/American Stroke Association Clinical Cardiology Council; American Heart Association/American Stroke Association Cardiovascular Radiology and Intervention Council; Atherosclerotic Peripheral Vascular Disease Working Group; Quality of Care Outcomes in Research Interdisciplinary Working Group. Guidelines for the early management of adults with ischemic stroke: a guideline from the American Heart Association/American Stroke Association Stroke Council, Clinical Cardiology Council, Cardiovascular Radiology and Intervention Council, and the Atherosclerotic Peripheral Vascular Disease and Quality of Care Outcomes in Research Interdisciplinary Working Groups: The American Academy of Neurology affirms the value of this guideline as an educational tool for neurologists. Circulation 2007;115(20):e478-e534

3 Donnan GA, Davis SM, Parsons MW, Ma H, Dewey HM, Howells DW. How to make better use of thrombolytic therapy in acute ischemic stroke. Nat Rev Neurol 2011;7(7):400-409

4 Sutherland BA, Minnerup J, Balami JS, Arba F, Buchan AM, Kleinschnitz C. Neuroprotection for ischaemic stroke: translation from the bench to the bedside. Int J Stroke 2012;7(5):407-418

5 Cho HJ, Kim YJ. Efficacy and safety of oral citicoline in acute ischemic stroke: drug surveillance study in 4,191 cases. Methods Find Exp Clin Pharmacol 2009;31(3):171-176

6 Yoshida H, Yanai H, Namiki Y, Fukatsu-Sasaki K, Furutani N, Tada N. Neuroprotective effects of edaravone: a novel free radical scavenger in cerebrovascular injury. CNS Drug Rev 2006;12(1):9-20

7 Matsukawa N, Yasuhara T, Hara K, et al. Therapeutic targets and limits of minocycline neuroprotection in experimental ischemic stroke. BMC Neurosci 2009;10:126

8 Zhang L, Chopp M, Meier DH, et al. Sonic hedgehog signaling pathway mediates cerebrolysin-improved neurological function after stroke. Stroke 2013;44(7):1965-1972

9 Durukan A, Tatlisumak T. Acute ischemic stroke: overview of major experimental rodent models, pathophysiology, and therapy of focal cerebral ischemia. Pharmacol Biochem Behav 2007;87(1):179-197

10 Fukuda S, Fini CA, Mabuchi T. Koziol JA, Eggleston LL Jr, del Zoppo GJ. Focal cerebral ischemia induces active proteases that degrade microvascular matrix. Stroke 2004;35(4):998-1004

11 Wang Q, Tang XN, Yenari MA. The inflammatory response in stroke. J Neuroimmunol 2007;184(1-2):53-68

12 Brundula V, Rewcastle NB, Metz LM, Bernard CC, Yong VW. Targeting leukocyte MMPs and transmigration: minocycline as a potential therapy for multiple sclerosis. Brain 2002;125(Pt 6) :1297-1308

13 Lampl Y, Boaz M, Gilad R, et al. Minocycline treatment in acute stroke: an open-label, evaluator-blinded study. Neurology 2007;69(14):1404-1410

14 Fagan SC, Waller JL, Nichols FT, et al. Minocycline to improve neurologic outcome in stroke (MINOS): a dose-finding study. Stroke 2010;41(10):2283-2287

15 Padma Srivastava MV, Bhasin A, Bhatia R, et al. Efficacy of minocycline in acute ischemic stroke: a single-blinded, placebo-controlled trial. Neurol India 2012;60(1):23-28

16 Amiri-Nikpour MR, Nazarbaghi S, Hamdi-Holasou M. Rezaei Y. An open-label evaluator-blinded clinical study of minocycline neuroprotection in ischemic stroke: gender-dependent effect. Acta Neurol Scand 2015;131(1):45-50

17 Kohler E, Prentice DA, Bates TR, et al. Intravenous minocycline in acute stroke: a randomized, controlled pilot study and meta-analysis. Stroke 2013;44(9):2493-2499

18 Heiss WD, Brainin M, Bornstein NM, Tuomilehto J, Hong Z; Cerebrolysin Acute Stroke Treatment in Asia (CASTA) Investigators. Cerebrolysin in patients with acute ischemic stroke in 
Asia: results of a double-blind, placebo-controlled randomized trial. Stroke 2012;43(3):630-636

19 Muresanu DF, Heiss WD, Hoemberg V, et al. Cerebrolysin and recovery after stroke (CARS): a randomized, placebo-controlled, double-blind, multicenter trial. Stroke 2016;47(1):151-159

20 Xue LX, Zhang T, Zhao YW, Geng Z, Chen JJ, Chen H. Efficacy and safety comparison of DL-3-n-butylphthalide and Cerebrolysin: effects on neurological and behavioral outcomes in acute ischemic stroke. Exp Ther Med 2016;11(5):2015-2020

21 Edaravone Acute Infarction Study Group. Effect of a novel free radical scavenger, edaravone (MCI-186), on acute brain infarction. Randomized, placebo-controlled, double-blind study at multicenters. Cerebrovasc Dis 2003;15(3):222-229

22 Sinha MK, Anuradha HK, Juyal R, Shukla R, Garg RK, Kar AM. Edaravone in acute ischemic stroke, an Indian experience. Neurol Asia 2009;14:7-10
23 Mitta M, Goel D, Bansal KK, Puri P. Edaravone - citicoline comparative study in acute ischemic stroke (ECCS-AIS) J Assoc Physicians India 2012;60:36-38

24 Gutiérrez-Fernández M, Rodríguez-Frutos B, Fuentes B, et al. CDP-choline treatment induces brain plasticity markers expression in experimental animal stroke. Neurochem Int 2012;60(3):310-317

25 Dávalos A, Alvarez-Sabín J, Castillo J, et al; International Citicoline Trial on acUte Stroke (ICTUS) trial investigators. Citicoline in the treatment of acute ischaemic stroke: an international, randomised, multicentre, placebo-controlled study (ICTUS trial). Lancet 2012;380(9839):349-357

26 Saver JL. Citicoline: update on a promising and widely available agent for neuroprotection and neurorepair. Rev Neurol Dis 2008;5(4):167-177

27 Ghosh S, Das SK, Nath T, Ghosh KC, Bhattacharyya R, Mondal GP. The effect of citicoline on stroke: a comparative study from the Eastern part of India. Neurol India 2015;63(5):697-701 